\title{
Run 16 Tandem gold performance in the injectors and possible improvement with AGS type 6:3:1 bunch merge in the Booster
}

K. Zeno

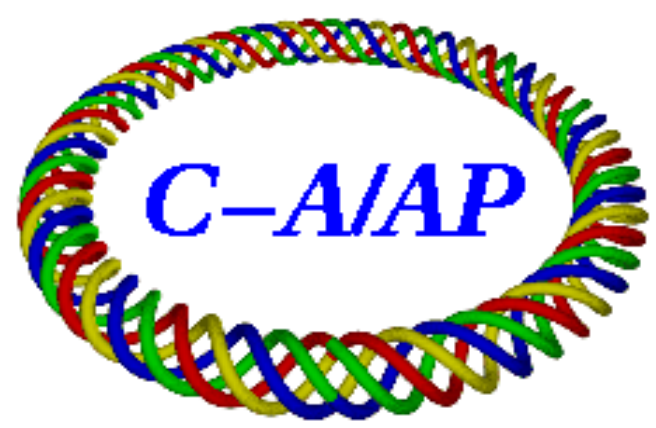

Collider-Accelerator Department Brookhaven National Laboratory Upton, NY 11973

\section{U.S. Department of Energy}

\section{Office of Science, Office of Nuclear Physics}

Notice: This document has been authorized by employees of Brookhaven Science Associates, LLC under Contract No. DE-SC0012704 with the U.S. Department of Energy. The United States Government retains a nonexclusive, paid-up, irrevocable, world-wide license to publish or reproduce the published form of this document, or allow others to do so, for United States Government purposes. 
Run 16 Tandem Gold Performance in the Injectors and Possible Improvement with an AGS Type 6:3:1 Bunch Merge in the Booster

Zeno, Keith

$10 / 21 / 2016$ 
During Run 16 the Tandem was used as the Gold pre-injector for a brief time so that RHIC could continue running while EBIS was down for repairs. Given the time constraints, the setup was largely derived from the EBIS Au setup. The EBIS Au setup used a 4:2:1 bunch merge in the Booster and a 12:6:2 bunch merge in the AGS. ${ }^{1}$ This note will describe the Tandem Au setup and compare it to that used for EBIS Au. The bunch merge in the Booster for Tandem Au did not work well, and it seems likely that the performance would've been significantly better if it did. An AGS type 6:3:1 merge in the Booster is described which might improve matters. ${ }^{2}$ Somewhat speculative estimates for the AGS bunch intensity and emittance, if that merge were successful in reducing the Booster extraction emittance to EBIS Au levels, are also given for several potential setups.

Using 6 Booster loads from the Tandem, the AGS bunch intensity at extraction reached about 2.5e9 ions with a longitudinal emittance $(\varepsilon)$ of about $0.59 \mathrm{eV} \cdot \mathrm{s} / \mathrm{n} .{ }^{3}$ Using 12 Booster loads from EBIS, the peak bunch intensity and $\varepsilon$ was about $3.1 \mathrm{e} 9$ ions and $0.75 \mathrm{eV} \cdot \mathrm{s} / \mathrm{n}$, respectively. A 6.4 sec supercycle was used for both at the time, but the Tandem Au supercycle (barring any potential issues with Tandem) could probably have been reduced to about 4.6 sec. ${ }^{4}$

\section{The Initial Setup}

Instead of 12 Booster transfers of 1 bunch each, with each set of 6 bunches merged into 1 AGS bunch, the Tandem setup used only 6 transfers with each set of 3 bunches merged into 1 AGS bunch. Yet, the AGS was initially configured for a 12:6:2 merge in the same way it was for EBIS. In that state the 12:6 merge was mixing full and empty buckets so that the emittance after that merge would be twice the emittance of the beam coming into it. This first 12:6 merge was unnecessary; it was initially there just as a matter of convenience.

Additionally, the fraction of the beam available at Booster extraction in the merged AGS bunches for the EBIS setup was nearly 50\%, but for the Tandem setup it was only about $31 \%{ }^{5}$ Figure 1 shows what a bunch looked like on the first turn in the AGS for both the EBIS and Tandem setups. ${ }^{6}$ The bunch in the Tandem case is much less uniform (the scales of the 2 pictures

\footnotetext{
${ }^{1}$ C-AD Note 571, "Overview and Analysis of the 2016 Gold Run in the Booster and AGS", K. Zeno, contains a detailed description of the Gold 12:6:2 setup used with the EBIS pre-injector during Run 16.

${ }^{2}$ K. Smith, "AGS Bunch Merging for Au2007" Presentation" gives an explanation of how the 6:3:1 type merge works in the AGS.

${ }^{3}$ This was found from the average of 4 flattop bunch widths measurements $([27.8+23+23.8+26.3] \mathrm{ns} / 4=25.225 \mathrm{~ns})$ made on May 5, 2016 using a synchrotron frequency of $94 \mathrm{~Hz}$ and an Rf frequency of $4.43701426 \mathrm{MHz}$. See Booster-AGS EBIS elog of May 5, 2016 for bunch width measurements.

${ }^{4}$ The EBIS Au supercycle was eventually reduced to $6.0 \mathrm{sec}$.

${ }^{5}$ See Overview and Analysis of the 2016 Gold Run in the Booster and AGS (pg. 41) for EBIS setup efficiency and the Booster-AGS-EBIS May 42016 (entries at 0425 and 0431) for Tandem.

${ }^{6}$ Booster-AGS EBIS elog of May 3, 2016, 2006 entry shows the Tandem Au first turn and Booster-AGS EBIS elog of May 19, 20161332 entry shows EBIS Au. Judging from archived data, the amplitude of Rf sine wave driving the quad pumping (which narrows the bunch at extraction) in the Tandem case appears to have been slightly greater $(18 \%)$ than in the EBIS case, though I'm not certain about this.
} 
are the same). There are only 2 pictures in the elog of the Tandem Au bunch on the first turn but there's little doubt that the bunch shape varied significantly from cycle to cycle. On this particular cycle the Tandem Au bunch doesn’t look much wider than the EBIS Au one, maybe 230 vs 200 ns.

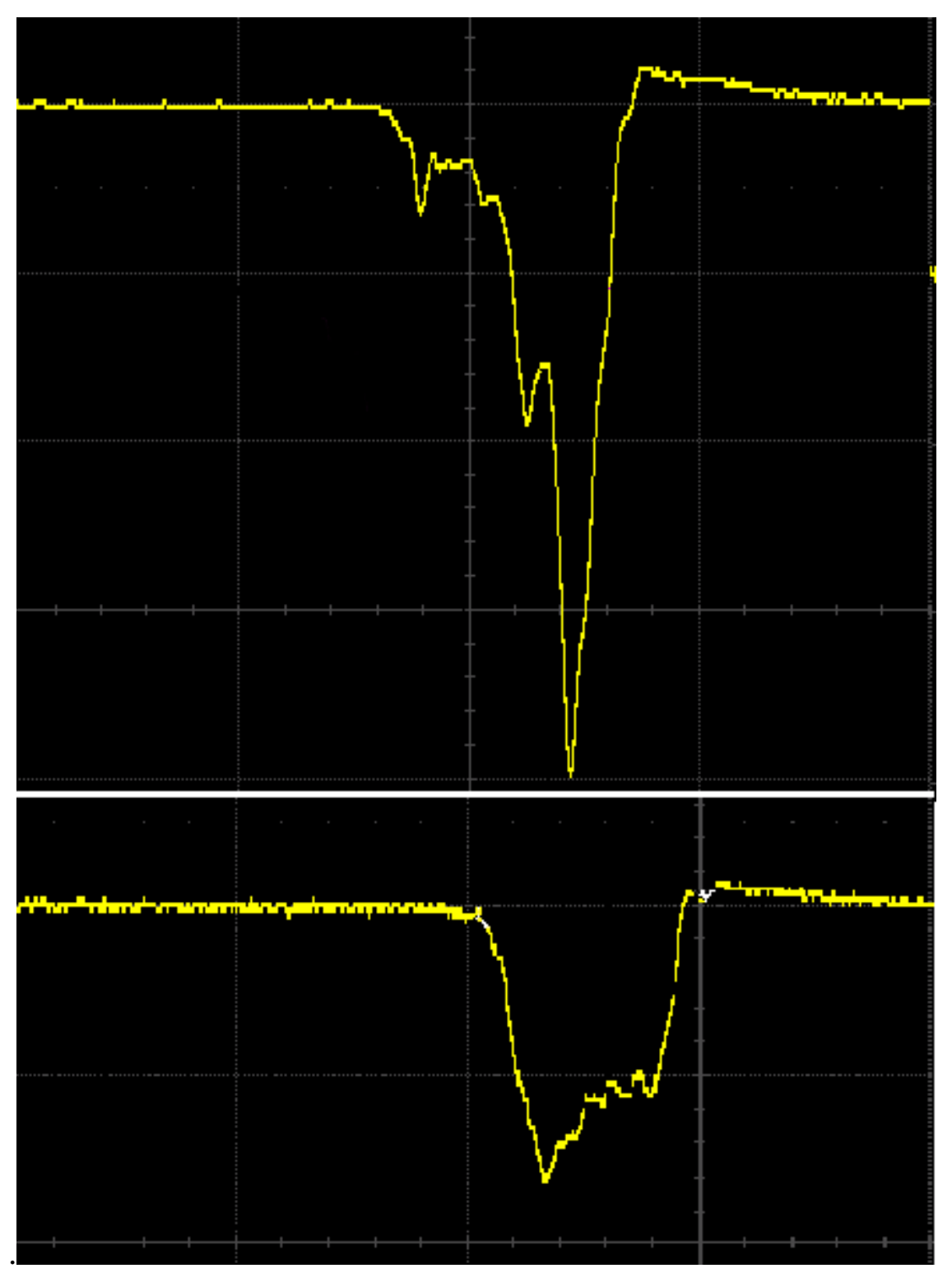

Figure 1: A typical bunch on the first turn in the AGS as viewed on the wall current monitor for the Tandem setup (top) and EBIS setup (bottom). Both are $50 \mathrm{mV} /$ box, $200 \mathrm{~ns} / \mathrm{div}$, and $50 \Omega$ termination. The scales of both the pictures are the same.

The main reason for the poor quality bunches is likely that the Booster merge for the Tandem setup did not work well. The Tandem setup requires 6 bunches to be merged into 1 instead of 4 . This is because the injection revolution frequency $\left(\mathrm{f}_{\text {rev }}\right)$ in the Tandem case is lower than in the EBIS case. The minimum Rf harmonic used to capture the beam at Booster injection 
is set by how low a frequency the Rf cavities can operate and that harmonic determines how many bunches will have to be merged.

There are 2 sets of $\mathrm{Rf}$ cavities in the Booster, the frequency range for one set (A3 and B3) is $350 \mathrm{kHz}$ to $5 \mathrm{MHz}$, and the frequency range for the other set (A6 and E6) is $770 \mathrm{kHz}$ to 1.45 $\mathrm{MHz}$. For EBIS, the injection $\mathrm{f}_{\text {rev }}$ is about $97 \mathrm{kHz}$, and it is about $66 \mathrm{kHz}$ for the Au delivered by Tandem. Consequently, $\mathrm{h}=4$ can be used for EBIS $(4.97 \mathrm{kHz}=388 \mathrm{kHz})$, but not for Tandem ( $4.66 \mathrm{kHz}=264 \mathrm{kHz}) . \mathrm{h}=5$ is also a bit below the lower limit for Tandem Au $(5.66 \mathrm{kHz}=330$ $\mathrm{kHz})$, but $\mathrm{h}=6$ is not $(6.66 \mathrm{kHz}=396 \mathrm{kHz})$, so $\mathrm{h}=6$ was used for capture and acceleration up to the merge energy.

In order to simplify the setup of Tandem Au, the field of the merge porch was kept the same as in the EBIS case. As with EBIS, two merges were used to produce one bunch. In the EBIS case a 4:2:1 merge is performed, but for Tandem a 6:2:1 merge had to be used. A6 and E6 are used to merge the 6 bunches into 2, and then A3 and B3 are used to merge those 2 bunches into one and accelerate it to extraction. ${ }^{7}$ Figure 2 shows how this 6:2:1 merge looked on a mountain range display. ${ }^{8}$ It is clear that the $6: 2$ merge does not work well, the 6 bunches appear to merge into 2 and then separate again. Figure 3 shows the 4:2:1 Booster merge used with EBIS Au for comparison. ${ }^{9}$ The frequency response of the Booster wall current monitor signal does not go as high as it does for the AGS WCM, which is why there is less bunch structure on it than there is on the AGS one (Figure 1).

EBIS delivers Au32+ and Tandem delivers Au31+. The Booster injection B $\rho$ is also significantly lower for Tandem. For EBIS the injection $\mathrm{B} \rho$ is about $1.25 \mathrm{~T} \cdot \mathrm{m}$ and for Tandem $\mathrm{B} \rho$ is $0.88 \mathrm{~T} \cdot \mathrm{m}$. There are a couple implications of these facts. First, since the injection rigidity is lower for Tandem, there was no room to increase the ramp rate, and the merge porch was already too short, the Booster cycle length had to be extended by 1/60 of a second to maintain the same extraction rigidity. Since there were only 7 cycles instead of the 13 used for EBIS operation, this could be done without exceeding the PPMR limit.

Secondly, with the same extraction $\mathrm{B} \rho$, the kinetic energy at extraction was about $6 \%$ lower with Tandem Au. This is relevant because the BtA foil's stripping efficiency is worse at lower energies and the bunches at extraction will be a bit wider for the same $\varepsilon$. It's not clear how large the reduction in stripping efficiency is, but any increase in the bunch length injecting into the $\mathrm{h}=24$ buckets used for the 12:6 merge wouldn't help. The difference in the Booster charge states also means that, for the same normalized transverse emittance, the transverse size will be

\footnotetext{
${ }^{7}$ A 6:3:1 merge could not be done because that would require $h=3$, not 2 , and the frequency for that would be about $1.65 \mathrm{MHz}$ which is above the upper limit for A6 and $\mathrm{E} 6$.

${ }^{8}$ Booster-AGS-EBIS elog April 3020161846 entry

9 Booster-AGS-EBIS elog March 16, 20161740 entry
} 
about 3\% larger. The AGS injection $\mathrm{B} \rho$ is also about 3\% lower for the Tandem setup. Figure 4 shows the state of the AGS in this configuration.

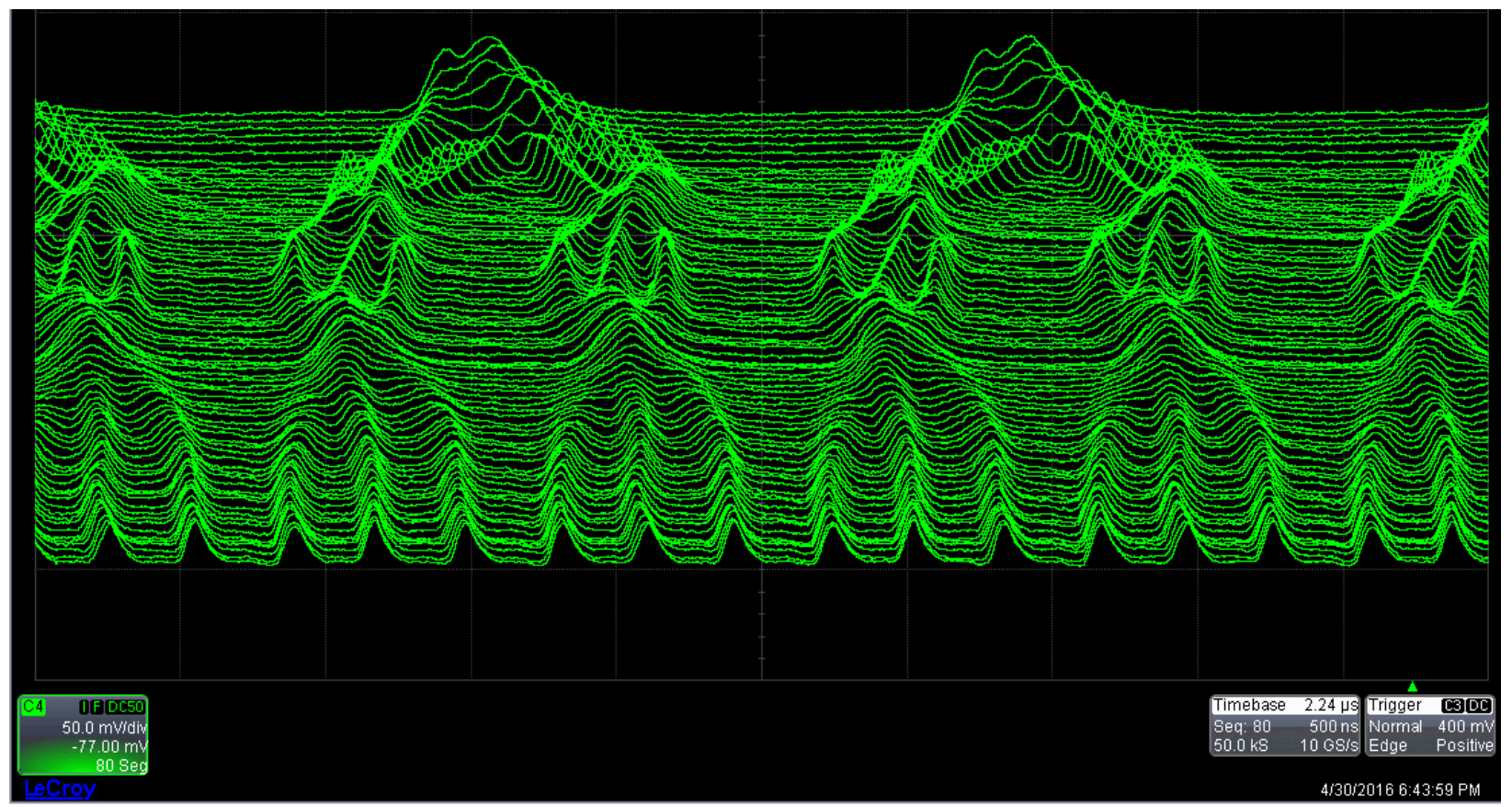

Figure 2: Mountain range display of the wall current monitor signal for the 6:2:1 Booster merge used for Tandem Au.

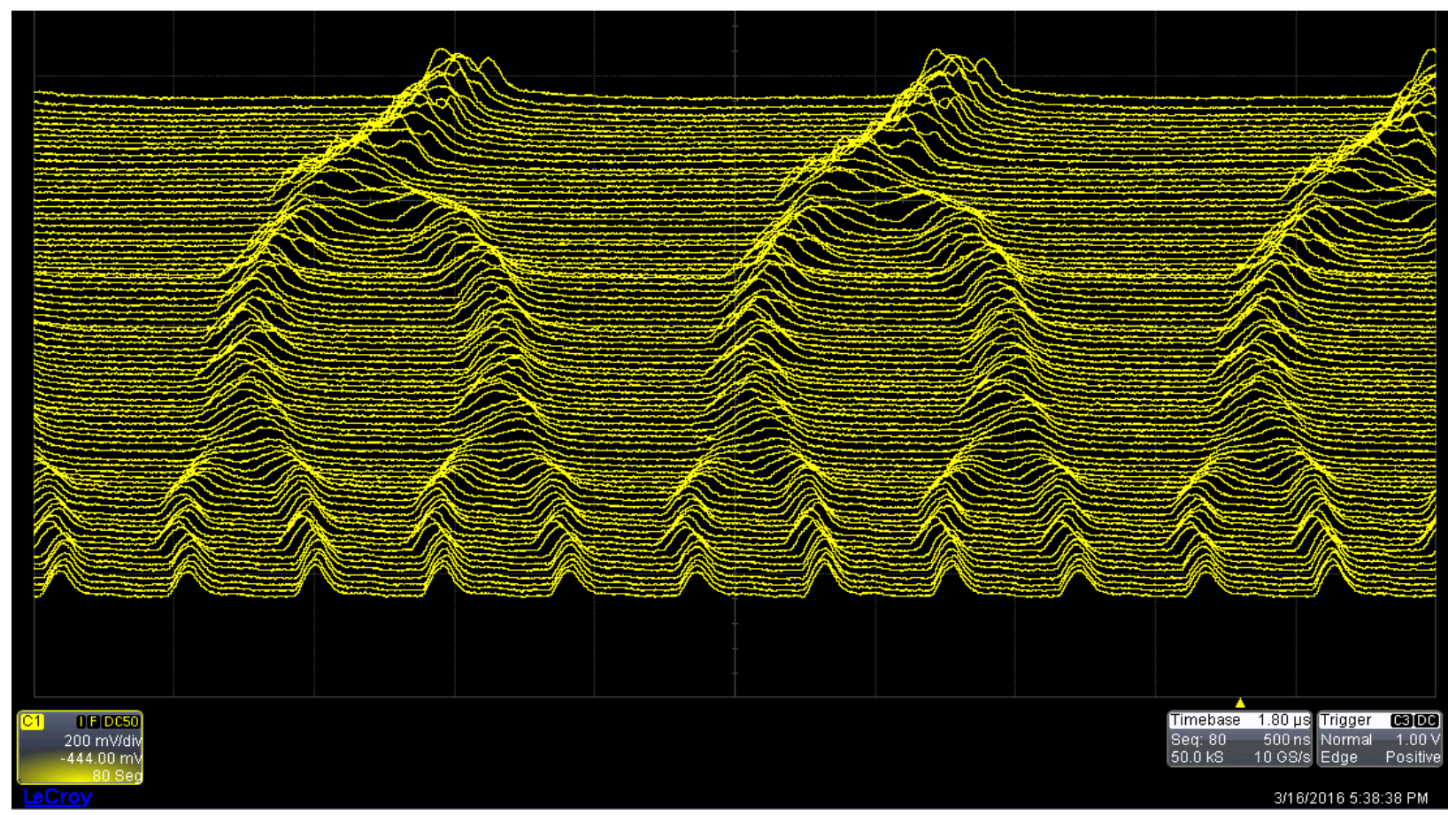

Figure 3: Mountain range display of the wall current monitor signal for the 4:2:1 Booster merge used for EBIS Au. 
The transfer efficiency (AGS just after last transfer/Booster late) at that time was about $40 \%(4.35 \mathrm{e} 9 / 10.8 \mathrm{e} 9)$ and the percentage of baby bunches was about $11 \% .{ }^{10}$ What appears to be a ( $6 \%$ ) capture loss is evident at the beginning of the ramp to the merge porch and an early acceleration loss is also visible. The main reason for the poor transfer efficiency (it's 53\% with the EBIS setup), most of the capture and early acceleration losses, and the relatively large percentage of baby bunches ( $4 \%$ for EBIS Au) is likely the larger $\varepsilon$ of the injected bunches compared to those for EBIS Au. The bunch intensity was in the 1.7e9 range for this Tandem intensity (about 24e9). For the same Booster late intensity (10.8e9 ions) using the EBIS setup the bunch intensity would be expected to be 2.6-2.7e9 ions.

\section{Removing the 12:6 merge in the AGS}

The beam is injected into $\mathrm{h}=24$ buckets, but the $\mathrm{h}=24$ bucket length at injection is $263 \mathrm{~ns}$. Without quad pumping the bunch is wider than that. It can be made narrower by increasing the quad pumping at Booster extraction but regardless the efficiency

(AGS bunch intensity/Booster late) could not be increased above $31 \%$ or so. As mentioned previously, the first AGS merge was unnecessary and also contributes to emittance growth because it mixes full and empty buckets. So, the AGS was configured without the first merge, and without that injection occurs into $\mathrm{h}=12$ buckets. Even the large bunches coming out of the Booster easily fit into $\mathrm{h}=12$ buckets.

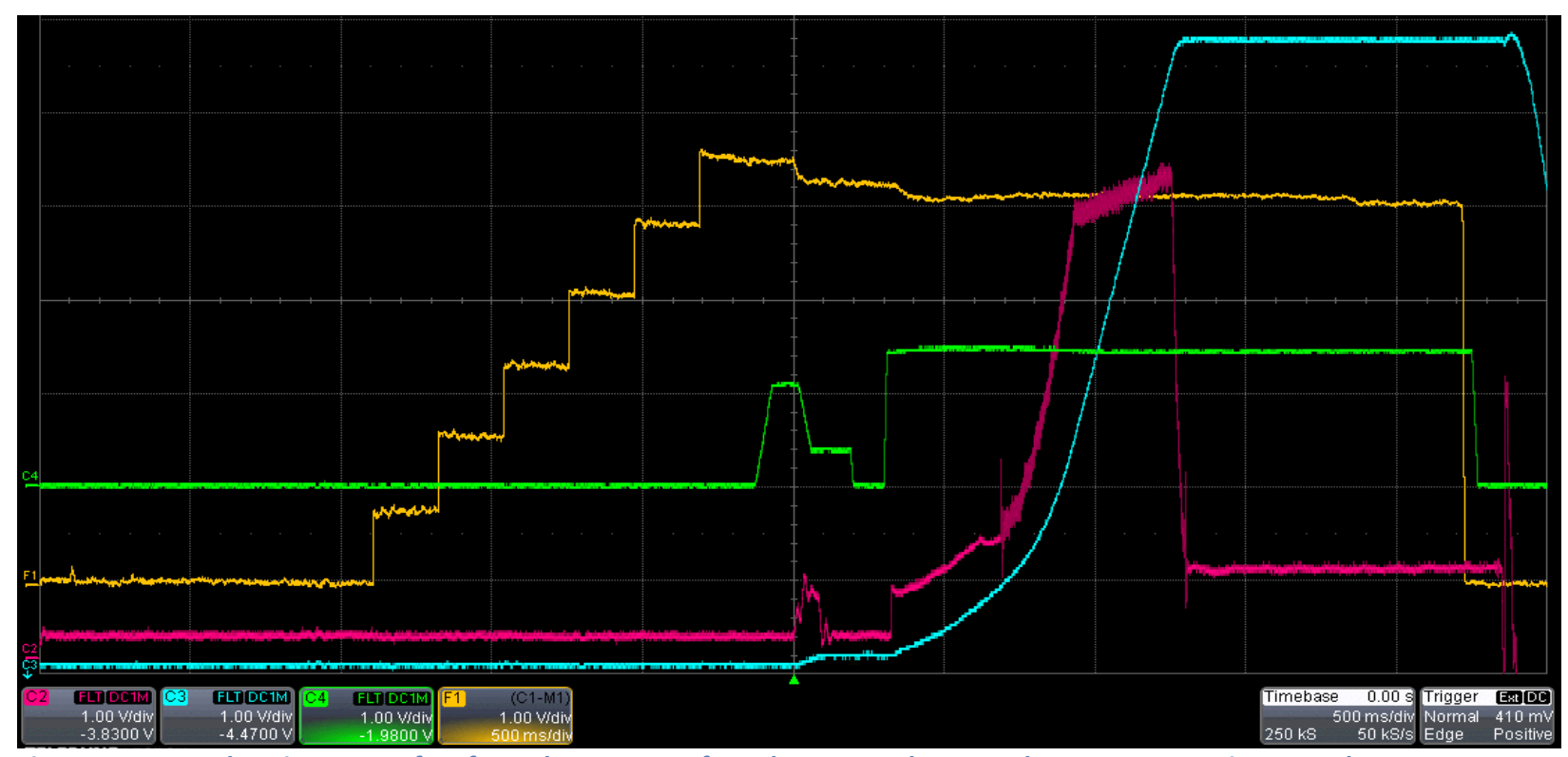

Figure 4: AGS cycle using 6 transfers from the Booster of Tandem Au31+ beam and a 12:6:2 merge in AGS. The orange trace is the AGS normalized transformer, the red is station I main magnet voltage, the blue is the AGS main magnet current, and the green is AGS Rf station K. The sweep speed is $500 \mathrm{~ms} /$ box. The trigger is at $2400 \mathrm{~ms}$ from At0. ${ }^{11}$

\footnotetext{
${ }^{10}$ The AGS intensity just after the last transfer was not available, but the AGS late intensity was (3.8e9). The scope picture was used together with that information to find the intensity at that time. The percentage of the baby bunches was found from the Booster-AGS-EBIS elog May 5, 20160006 entry.

${ }^{11}$ Booster-AGS-EBIS elog May 4, 2016, 0314 entry
} 
Figure 5 shows the state of the AGS after that change. This change brought the transfer efficiency up to about 46\% (5.43e9/11.7e9), and also reduced the capture and early acceleration losses. ${ }^{12}$ The baby bunch percentage in this state was about $4 \% .{ }^{13}$ The late intensity was about $5.1 \mathrm{e} 9$, which would give a bunch intensity of (1-0.04) $5.1 \mathrm{e} 9 / 2=2.45 \mathrm{e} 9$ ions. The intensity of a bunch around this time was also measured to be $2.5 \mathrm{e} 9$ ions using the wall current monitor. ${ }^{14}$

Although one could argue that the reason that the transfer efficiency is still lower than in the EBIS case is because of the lower transfer energy, it seems more likely to me that it is mainly due to the poorer quality bunches. A large $\Delta \mathrm{p} / \mathrm{p}$ will tend to reduce the transfer efficiency because of the limited momentum aperture. Although the quad pumping at Booster extraction, which is used to reduce the bunch width at the expense of $\Delta \mathrm{p} / \mathrm{p}$, was turned off once the 12:6 merge was removed the general spikiness of the bunch shape likely indicates a relatively large number of particles with a large $\Delta \mathrm{p} / \mathrm{p}$ in the transferred bunches.

It's also possible to estimate the injected bunch $\varepsilon$ from the flattop measurement mentioned earlier that was done in this state $(0.59 \mathrm{eV} \cdot \mathrm{s} / \mathrm{n})$. Assuming $45 \% \varepsilon$ growth during the main acceleration ramp and no $\varepsilon$ growth during the merge, would give an injected bunch $\varepsilon$ of $(1 / 1.45) \cdot(0.59 \mathrm{eV} \cdot \mathrm{s} / \mathrm{n}) / 3=0.136 \mathrm{eV} \cdot \mathrm{s} / \mathrm{n}$ after equilibration but before the merge. ${ }^{15}$ Direct measurements with EBIS Au gave an $\varepsilon$ of $0.088 \mathrm{eV} \cdot \mathrm{s} / \mathrm{n}$ for those bunches. ${ }^{16}$ And the fact that removing the 12:6 merge had such a large effect on the efficiency is also indicative of larger injected bunches.

It's also true that when we regularly ran Tandem Au the extraction energy was essentially the same as it is here and the transfer efficiency was something like $57 \% .{ }^{17}$ In that case there were 6 (unmerged) bunches transferred per Booster cycle so the maximum $\Delta \mathrm{p} / \mathrm{p}$ of the transferred bunches was likely significantly smaller.

For the efficiency measurement without the 12:6 merge the Booster late was higher (11.7e9 vs. 10.8e9), and if it were the same one would expect the AGS intensity after the last transfer to be $0.46 \cdot 10.8 \mathrm{e} 9=5.0 \mathrm{e} 9$ ions. Using the AGS efficiency after the change,

\footnotetext{
${ }^{12}$ The quad pumping at Booster extraction was also turned off once the 12:6 merge was removed.

${ }^{13}$ This was measured from logged AGS transformer data during a fill (May 6, 2016 2151)

${ }^{14}$ See Booster-AGS-EBIS May 520161723 entry.

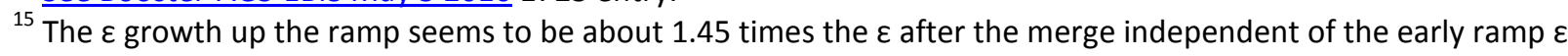
(see C-AD Note 571, "Overview and Analysis of the 2016 Gold Run in the Booster and AGS", K. Zeno, pgs. 34-35) and the $\varepsilon$ growth for the 6:2 merge was measured in the EBIS Au case and was negligible (pg. 30 of note 571). ${ }^{16}$ See C-AD Note 571, "Overview and Analysis of the 2016 Gold Run in the Booster and AGS", K. Zeno, pg. 29.

${ }^{17}$ See April 272011 Booster-AGS-Au elog, L. Ahrens' entry at 1247 states that, in terms o the Rf frequency, the AGS injection revolution frequency was $3.80245 \mathrm{MHz}$ for foil 5 . I believe this foil is the same type as the one we use now. This is $h=24$, so frev $=158435 \mathrm{kHz}$. This run frev was $158225 \mathrm{kHz}$, so the transfer energy was about $0.3 \%$ higher then or essentially the same. The Booster-AGS-Au elog April 2220111833 entry shows a transfer efficiency (AGS CBM/Booster Late) of 56.95\%. This was for 4 not 6 transfers, but that wouldn't be expected to make a large difference.
} 
(AGS Late/AGS just after the last transfer) $=5.1 / 5.43=0.94$, would give an AGS late intensity of 5.0e9-0.94=4.7e9 ions, and the bunch intensity would be $(1-0.04) \cdot 4.6 e 9 / 2=2.26 e 9$. So, the removal of the 12:6 merge increased the bunch intensity by about 33\% (2.26e9/1.7e9) and brought (AGS bunch intensity/Booster late) up to about $42 \%$.

Running with 6 instead of 4 pulses from Tandem was uncharted territory so it was not clear how high the Tandem intensity (a.k.a. Booster input) would go but it did reach 30e9 ions before the removal of the 12:6 merge. At that time the Booster efficiency (Booster Late/Booster Input) was about (14e9/30e9)=47\%. The Booster efficiency is a function of the input intensity for Tandem $\mathrm{Au}$, and there is plenty of data from running with 4 Tandem pulses which can be used to estimate what the efficiency would be for 30e9 if the Booster were fully optimized. Assuming all the Tandem pulses are of equal intensity, a single Tandem pulse is $30 \mathrm{e} 9 / 6=5 \mathrm{e} 9$, and 4 pulses of 5e9 each is 20e9. A 4 pulse input of 20e9 would give a Booster late of $10.5 \mathrm{e} 9 \pm 0.7 \mathrm{e} 9$ and an efficiency of $53 \%$ if the Booster were fully optimized, which corresponds to $15.7 \pm 1 \mathrm{e} 9$ for 6 pulses. ${ }^{18}$ Fully optimizing the Booster for Tandem Au typically takes on the order of weeks not days, and it also requires a good deal of attention to maintain it.

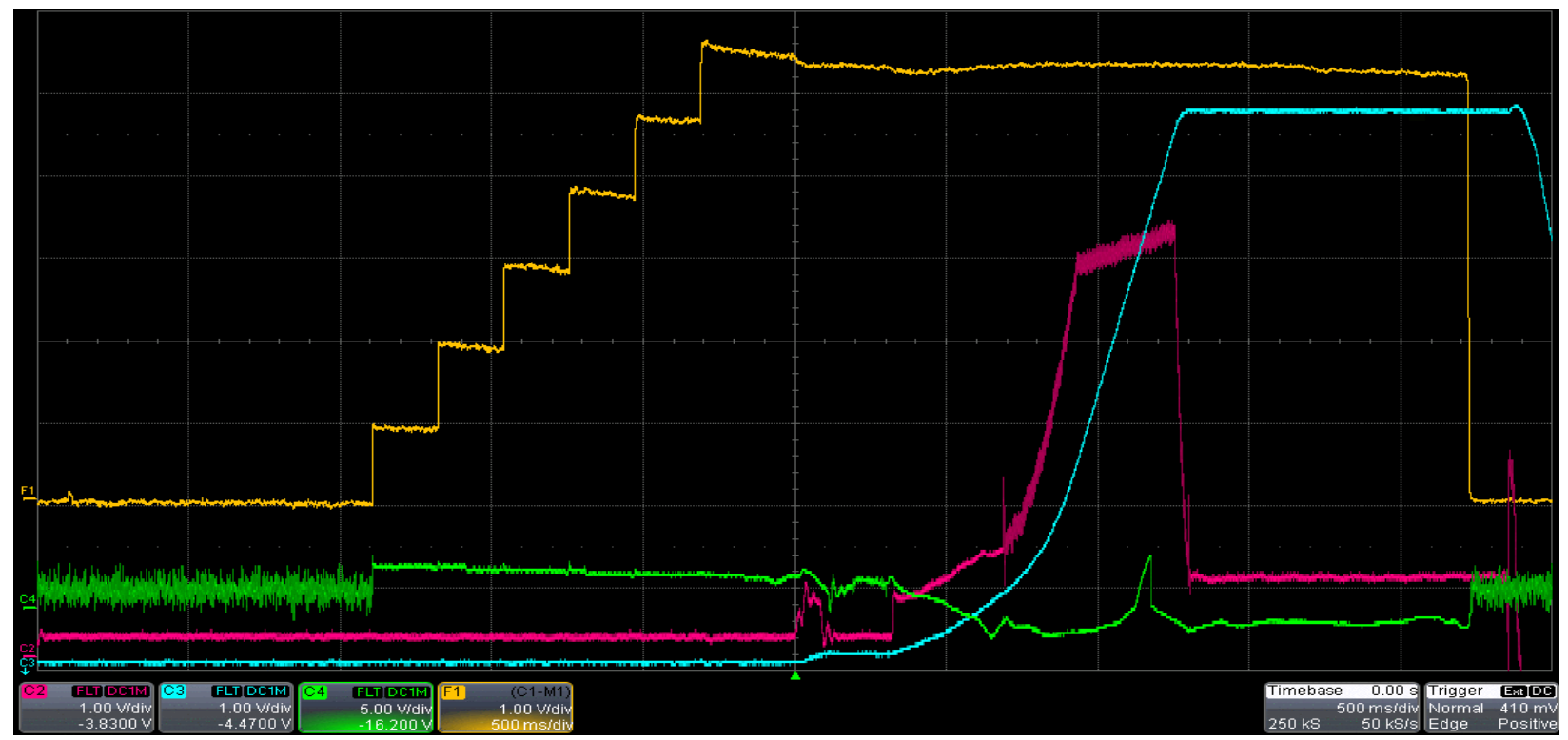

Figure 5: AGS cycle after the removal of the 12:6 merge and switch from $\mathrm{h}=\mathbf{2 4}$ to $\mathrm{h}=\mathbf{1 2}$ buckets at injection. The orange trace is the AGS normalized transformer, the red is station I main magnet voltage, the blue is the AGS main magnet current, and the green is a horizontal BPM signal. The sweep speed is $500 \mathrm{~ms} /$ box. The trigger is at $2400 \mathrm{~ms}$ from At0. ${ }^{19}$

\footnotetext{
${ }^{18}$ See Booster-AGS-EBIS 2007 elog Jun 81829 entry. In that reference, the top set of curves shown in the rightmost plot indicate the normal range for Booster late as a function of Booster input. This plot is for 4 Tandem pulses, so the equivalent of $30 \mathrm{e} 9$ input is $(4 / 6) \cdot 30 \mathrm{e} 9=20 \mathrm{e} 9$. The curves indicate that a typical Booster late for that input is 10.5e9. This is then multiplied by $6 / 4$ to get the expected Booster late for $30 \mathrm{e} 9$ with 6 pulses,

$(6 / 4) \cdot 10.5 \mathrm{e} 9=15.75 \mathrm{e}$. These curves are likely for a $900 \mu \mathrm{s}$ Tandem pulse since according to an archive at the time the pulse width appears to have been that. The pulse width when a Booster input of $30 \mathrm{e} 9$ ions was present this run was also $900 \mu$ s according to an archive around that time.

${ }^{19}$ Booster-AGS-EBIS elog May 5, 2016, 1718 entry
} 
If the quality of the bunches coming out of the Booster could be improved the transfer efficiency would likely increase and the longitudinal $\varepsilon$ would decrease. The transfer efficiency using the 8:4:2 setup, which uses $\mathrm{h}=16$ buckets, is about $56 \%$. If the extraction $\varepsilon$ and energy were the same as for the EBIS Au setup, then this is about the transfer efficiency one would expect.

Since there are only 3 bunches being merged into 1 , if the bunches coming from the Booster were the same size as in the EBIS case, the $\varepsilon$ after the AGS merge should be about $0.30 \mathrm{eV} \cdot \mathrm{s} / \mathrm{n}$ and it's unlikely that there would be any baby bunches. ${ }^{20}$ The $\varepsilon$ on flattop would be expected to be about $1.45 \cdot(0.30 \mathrm{eV} \cdot \mathrm{s} / \mathrm{n})=0.44 \mathrm{eV} \cdot \mathrm{s} / \mathrm{n} .{ }^{21}$ For the $8: 4: 2$ setup the efficiency (AGS Late/Booster Late) is about 52\%, so barring any intensity related effects in the AGS, such as instabilities, the bunch intensity would be about $0.52 \cdot 15.7 \mathrm{e} 9 / 2=4.1 \mathrm{e} 9 .^{22}$ There would be about 8e9 ions at the end of the injection porch, and there were some instabilities with the 12:6:2 setup where the intensity at the end of that porch was only about 6 to $6.5 \mathrm{e} 9$ ions. It was relatively easy to prevent them from occurring by adjusting the octupoles there, but it would likely become more difficult at higher intensities. ${ }^{23}$

\section{An AGS Type 6:3:1 Merge in the Booster}

It appears that what stands in the way of reaching this intensity and longitudinal $\varepsilon$ is the quality of the merge in the Booster. But it may be possible to do a 6:3:1 merge in the Booster like the one in the AGS which has been shown to preserve emittance if the merge porch is lowered from about $5500 \mathrm{~g}$ to $4000 \mathrm{~g}$. The 4:2:1 merge used with EBIS has one less step than this type of 6:3:1 merge, so the porch needs to be longer. Since there are only 7 cycles, it's possible to extend this lower merge porch from the length used for the 4:2:1 merge ( $\sim 17 \mathrm{~ms}$ ) to $33.7 \mathrm{~ms}$ by increasing the Booster cycle length another $1 / 60$ of a second to $233.3 \mathrm{~ms}$ without exceeding the PPMR limit in the Superman calculation. ${ }^{24}$

\footnotetext{
${ }^{20}$ The $\varepsilon$ of the unmerged Tandem beam in the Booster appears to have been significantly smaller than for EBIS. In 2007 the 6 bunch $\varepsilon$ at Booster extraction was measured to be $(0.082 \mathrm{eV} \cdot \mathrm{s} / \mathrm{n}) / 1.8=0.046 \mathrm{eV} \cdot \mathrm{s} / \mathrm{n}$ (see $\mathrm{L}$. Ahrens, et. al., "Setup and Performance of the RHIC Injector Accelerators for the 2007 Run with Gold lons", pg. 1864, Proceedings of PAC07, Albuquerque, New Mexico, USA). A similar measurement with EBIS Au from Run 14 gives $0.08 \mathrm{eV} \cdot \mathrm{s} / \mathrm{n}$ (see K. Zeno, "Longitudinal Emittance Measurements in the Booster and AGS during the $2014 \mathrm{Gold}$ Run”, pg. 7, Table IV, CAD Note 523, August 2014). So, the $\varepsilon$ of the injected beam might be significantly smaller than in the EBIS case if the merge worked well.

${ }^{21}$ A typical bunch on the injection porch with the 8:4:2 setup was measured to be $0.10 \mathrm{eV} \cdot \mathrm{s} / \mathrm{h}$ and the $\varepsilon$ growth up the ramp seems to be about 1.45 times the $\varepsilon$ after the merge independent of the early ramp $\varepsilon$ (see C-AD Note 571 , "Overview and Analysis of the 2016 Gold Run in the Booster and AGS", K. Zeno, pgs. 29 and 34-35).

${ }^{22}$ See for example, Booster-AGS-EBIS Jan 212016 elog 1818 entry where the 8:4:2 transfer efficiency (AGS CBM/Booster Late) is $56 \%$ and (AGS Late/Booster Late) is $52 \%$.

${ }^{23}$ See Booster-AGS-EBIS Feb 262016 and Booster-AGS-EBIS March2, 2016 elog.

${ }^{24}$ The PPMR limit is satisfied using the calculation in Superman but a supercycle with 7 of these magnet cycles pulsing hasn't been run. See Booster-AGS-EBIS 2017 elog Oct 41506 entry.
} 
Figure 6 shows how the 4:2:1 merge is accomplished ${ }^{25}$, and Figure 7 shows this 6:3:1 merge schematically. Figure 7 is just intended to show how the different cavities would be used and the general timing of those cavities, the voltage amplitudes shown are rather arbitrary. With the lower porch, it looks like one of the narrow frequency range cavities (A6/E6) can be used for $h=2$ and the other for $h=3$, and the porch would still be high enough for A3/B3 to operate on $h=1$. The figure shows the approximate frequencies that the cavities would run at. Only one narrow frequency range cavity is required for the 4:2:1 merge, so one might hope that there is enough voltage for one of these cavities to be used for $h=3$ and the other for $h=2$.

Of course, it is far from certain this merge would work as well as the 4:2:1 merge does. The main issue appears to be if there is enough time for it. The 4:2:1 merge has about half the time that this would have, and it doesn't appear that there is much $\varepsilon$ growth from it. ${ }^{26}$ The $4: 2: 1$ merge basically has 3 steps to it:

1) $\mathrm{h}=4$ falls and $\mathrm{h}=2$ rises

2) Allow some time for A3/B3 to switch from $h=4$ to 1

3) $\mathrm{h}=2$ falls as $\mathrm{h}=1$ rises

And for the 6:3:1 merge the steps are:

1) $h=6$ falls and $h=3$ rises

2) $h=3$ falls to about $1 / 3$ of its peak value (this also provides time for $A 3 / B 3$ to switch from $h=6$ to 1 )

3) $\mathrm{h}=1$ and $\mathrm{h}=2$ rise

4) $\mathrm{h}=2$ and 3 fall

So, the 6:3:1 merge has 4 steps and 4:2:1 has 3 steps, given that the 6:3:1 merge would have twice as much time one might hope it would perform as well as the 4:2:1 merge, but that remains to be seen.

It was also possible to construct a Booster magnet cycle with a peak rigidity that is a factor of 32/31 higher than the present EBIS Au cycle and is still $233.3 \mathrm{~ms}$ long. It does not exceed the PPMR limit in the calculation for seven cycles. This cycle also has the same early ramp as the Tandem Au cycle used years ago to fill RHIC (which the one in Run 16 did not have). Although this cycle should provide the same extraction energy as for EBIS Au, the rigidity is higher than we have run and the strength of the F3 kicker, which already runs at full current, might become a factor.

\footnotetext{
${ }^{25}$ The 6:2:1 merge used in Run 16 was basically the same except the A3/B3 harmonic accelerating up to the merge was 6.

${ }^{26}$ See C-AD Note 571, “Overview and Analysis of the 2016 Gold Run in the Booster and AGS", K. Zeno, pg. 28 where it is mentioned that measurements indicate the growth may be on the order of $10 \%$ for the 4:2:1 merge. The 6:3:1 AGS merge is shown on page 18 of that note (in figure 10).
} 


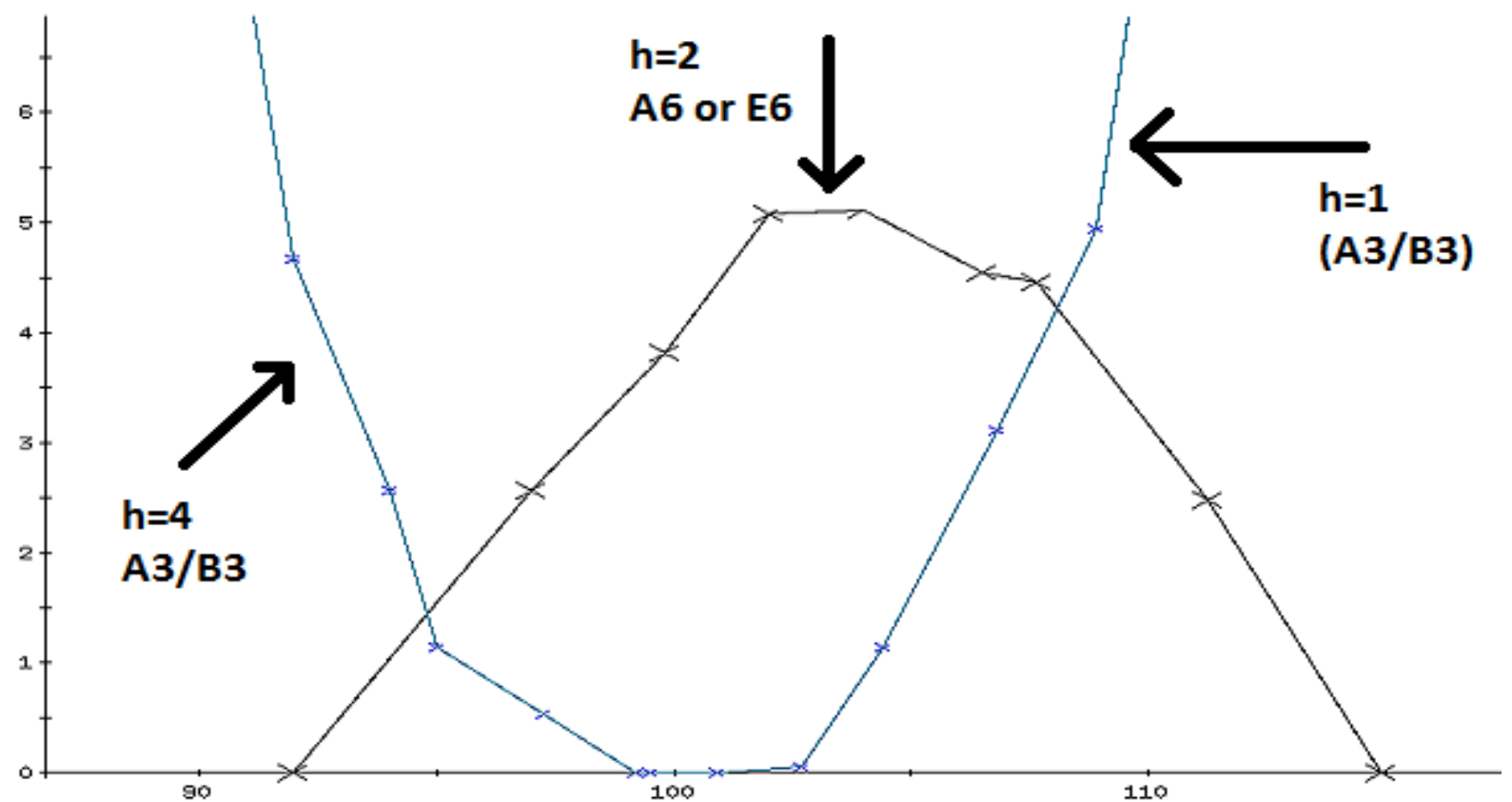

Figure 6: $\mathbf{R f}$ gap voltages, harmonics, and cavities involved in the standard 4:2:1 Booster merge used for EBIS Au. The $x$-axis is $\mathrm{ms}$ from Bt0 and the $y$-axis is the voltage reference. The $h=2$ cavity has 2 gaps, and A3 and B3 have 1 gap. So, since both A3 and $B 3$ are used for $h=4$ and $h=1$ the relative voltages here should be correct.

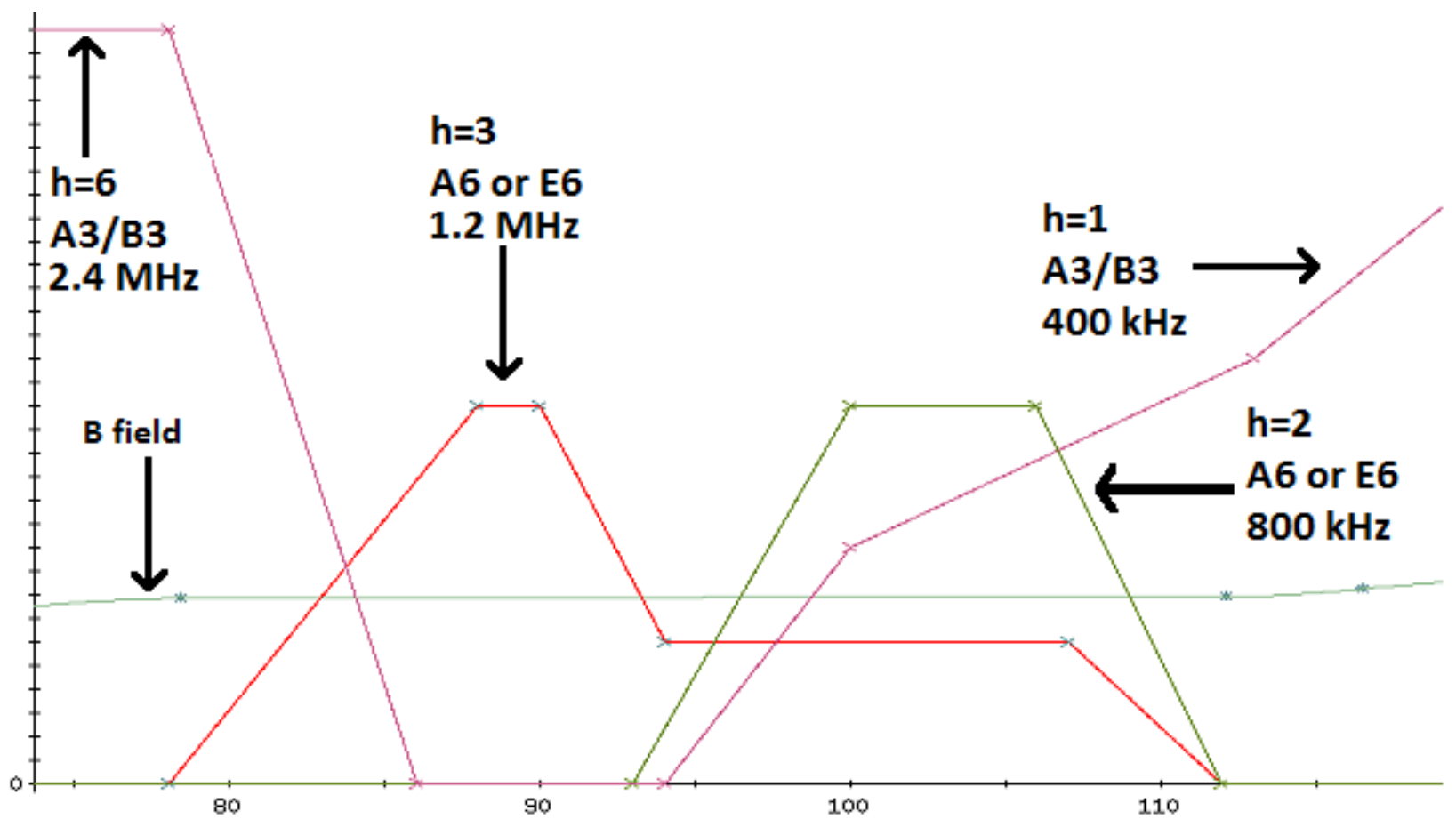

Figure 7: Schematic of an AGS type 6:3:1 merge in the Booster showing the harmonics, cavities, and frequencies involved. The Booster main magnet field is also included to show the porch. The $x$-axis is time in ms from Bt0, the y-axis is voltage. The voltages shown are arbitrary; the plot is only intended to be understood schematically. 


\section{Some Other Possible Scenarios}

The bunch intensity and $\varepsilon$ estimates for the following scenarios assume the same Booster extraction $\varepsilon$ as in the EBIS Au case, and no deterioration in the transfer efficiency from the slightly lower Booster extraction energy. Among other things, they also assume no intensity related effects in the AGS and $45 \% \varepsilon$ growth from the AGS main magnet pulsed voltage bank:

1) If Tandem delivered only 4 pulses, they could be merged into 1 using a 4:2:1 merge in the AGS (inject into $h=16$, use $h=8$ and 4 to merge into 1 , and accelerate on $h=12$ ). In this case there would only be one bunch per AGS cycle. Years ago, when Tandem was the Au pre-injector, 4 pulses were used and the Booster late intensity was typically higher than 12.0e9 ions. So, the bunch intensity would be in the 6e9 range and the $\varepsilon$ at the nominal extraction energy would be expected to be about $1.45 \cdot 0.4 \mathrm{eV} \cdot \mathrm{s} / \mathrm{n}=0.58 \mathrm{eV} \cdot \mathrm{s} / \mathrm{n}$. In the case of an AGS extraction energy low enough that it doesn't require the pulsed voltage bank (i.e.-for a very low energy RHIC run), the $\varepsilon$ would be expected to be about $0.4 \mathrm{eV} \cdot \mathrm{s} / \mathrm{n}$. The AGS cycle length could be as short as about $4.2 \mathrm{sec}$.

2) It would also be possible to merge all 6 Booster transfers into 1 AGS bunch by restoring the 12:6 (a.k.a. 6:3) AGS merge and filling 6 consecutive $h=24$ buckets at injection. The injected bunches may have more difficulty fitting into the $\mathrm{h}=24$ buckets than EBIS Au because the transfer energy is $6 \%$ lower. Neglecting this, it would be expected to provide

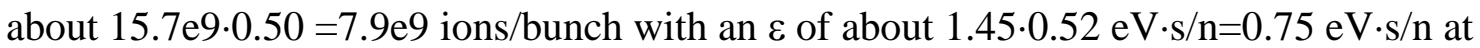
the nominal AGS extraction energy. ${ }^{27}$ In the case of an AGS extraction energy low enough that it doesn't require the AGS main magnet pulsed voltage bank the $\varepsilon$ would be expected to be about $0.52 \mathrm{eV} \cdot \mathrm{s} / \mathrm{n}$. The AGS cycle length could be as short as about 4.6 sec.

3) If Tandem could deliver 8 pulses then an 8:4:2 merge in the AGS could be used to provide 2 bunches per AGS cycle. The peak field of the Booster magnet cycle would have to be lowered about 5\% to stay below the PPMR limit. This would likely reduce the transfer efficiency. Since the Tandem has never delivered 8 pulses in a supercycle I'm unable to give an estimate for the bunch intensity, but the $\varepsilon$ would be the same as for the 4:2:1 merge (case 1). The AGS cycle length could be as short as about 5.2 sec.

4) With 8 Tandem pulses the injected bunches could perhaps be merged into 1 in the AGS using the hypothetical 8:4:2:1 merge discussed in C-AD Note $571 .{ }^{28}$ Again, the peak Booster field would have to be lowered about $5 \%$. With the lower Booster extraction energy, fitting the injected bunches into $\mathrm{h}=24$ buckets would likely become more of an

\footnotetext{
${ }^{27}$ See C-AD Note 571, "Overview and Analysis of the 2016 Gold Run in the Booster and AGS", K. Zeno, Table VI on pg. 42 where it is indicated that the e for the 12:6:2 merge in AGS was $0.52 \mathrm{eV} \cdot \mathrm{s} / \mathrm{n}$ just before the transfer to the pulsed $\mathrm{V}$ bank (item 11).

${ }^{28}$ See C-AD Note 571, "Overview and Analysis of the 2016 Gold Run in the Booster and AGS", K. Zeno, pgs. 37-41 (where it's referred to as the 16:8:4:2 merge because 16 bunches are being merged into 2 ). The frequencies of the specialized AGS cavities used for merges (KL and L10) would also have to be lowered from where they are now.
} 
issue. The $\varepsilon$ at the nominal flattop energy would be expected to be around $1.13 \mathrm{eV} \cdot \mathrm{s} / \mathrm{n}$, and at an energy below where the use of the pulsed voltage bank is necessary it would be about $0.79 \mathrm{eV} \cdot \mathrm{s} / \mathrm{n}$. The AGS cycle length could be as short as about $5.2 \mathrm{sec}$.

At the risk of getting carried away, there are other possibilities too which don't provide as much bunch intensity as the preceding ones but would have more bunches per AGS cycle and smaller $\varepsilon$.

5) Use 6 Tandem pulses but only do a 6:3 merge in the AGS ( $\mathrm{h}=24$ to 12 ). Then there would be 3 bunches, each with $0.52 \cdot 15.7 e 9 / 3=2.7 e 9$ ions per bunch and with an $\varepsilon$ of about $1.45 \cdot 2 \cdot 0.10 \mathrm{eV} \cdot \mathrm{s} / \mathrm{n}=0.29 \mathrm{eV} \cdot \mathrm{s} / \mathrm{n}$ at the nominal extraction energy. The AGS cycle length would be slightly shorter than case 2 because there is no AGS merge porch required, maybe about $4.4 \mathrm{sec}$.

6) Use 4 Tandem pulses but only do a 4:2 merge in the AGS ( $\mathrm{h}=24$ to 12 ). Then there would be 2 bunches, each with $0.52 \cdot 12 \mathrm{e} 9 / 2=3.1 \mathrm{e} 9$ ions per bunch and with an $\varepsilon$ of $1.45 \cdot 2 \cdot 0.10 \mathrm{eV} \cdot \mathrm{s} / \mathrm{n}=0.29 \mathrm{eV} \cdot \mathrm{s} / \mathrm{n}$ at the nominal extraction energy. This gives a higher bunch intensity than case 5 because the Tandem intensity would likely be higher with only 4 pulses. The AGS cycle length would be about 4.0 sec.

7) Use 4 Tandem pulses with no merge in the AGS (inject and accelerate on $h=12$ ). Then there would be 4 bunches each containing $0.52 \cdot 12.0 \mathrm{e} 9 / 4=1.6 \mathrm{e} 9$ ions and each with an $\varepsilon$ of about $0.15 \mathrm{eV} \cdot \mathrm{s} / \mathrm{n}$ at the nominal extraction energy. This $\varepsilon$ is smaller than during previous Tandem Au running with one Booster load going into 1 AGS bunch $(\sim 0.23 \mathrm{eV} \cdot \mathrm{s} / \mathrm{n})$ presumably because there would not be the $\varepsilon$ growth before the pulsed bank since there is no AGS merge. ${ }^{29}$ The AGS cycle length could be about $3.8 \mathrm{sec}$ because there's no time needed for an AGS merge. This would also work for more than 4 Tandem pulses.

8) Use 8 Tandem pulses and only do an 8:4 merge ( $\mathrm{h}=24$ to 12). That would produce 4 AGS bunches each with a nominal flattop $\varepsilon$ of $0.29 \mathrm{eV} \cdot \mathrm{s} / \mathrm{n}$. The bunch intensity would probably be lower than in case 5 because the Tandem intensity would probably be lower and because the Booster extraction field would have to be $5 \%$ lower as well. The AGS cycle length could be as short as $5.2 \mathrm{sec}$.

Figure 8 graphically summarizes these scenarios. Note that Tandem has not yet delivered 8 pulses in a supercycle so it is not even known if they can and the 8 to 1 merge in the AGS (case 4, upper right corner) has never even been done with EBIS Au. In one shape or form the other merges have been done with EBIS Au, and so should work for Tandem as well. Scenarios 5, 6, and 8, where injection occurs into $\mathrm{h}=24$ buckets and which only do a 2:1 type merge using $\mathrm{h}=12$ could use $\mathrm{h}=16$ and 8 instead for the 2:1 merge and accelerate with $\mathrm{h}=12$. That might be preferable since fitting an injected bunch into an $\mathrm{h}=24$ bucket is often an issue.

\footnotetext{
${ }^{29}$ See C-AD Note 571 , K. Zeno, bottom of pg.4 and top of pg. 5. The $\varepsilon$ growth through the BtA foil would also be less because there is only one bunch instead of 6 but these estimates assume the same injection $\varepsilon$ as in the EBIS Au case which is about the same as it was for Tandem Au.
} 


\section{Possible scenarios using 4,6 and 8 Tandem pulses}

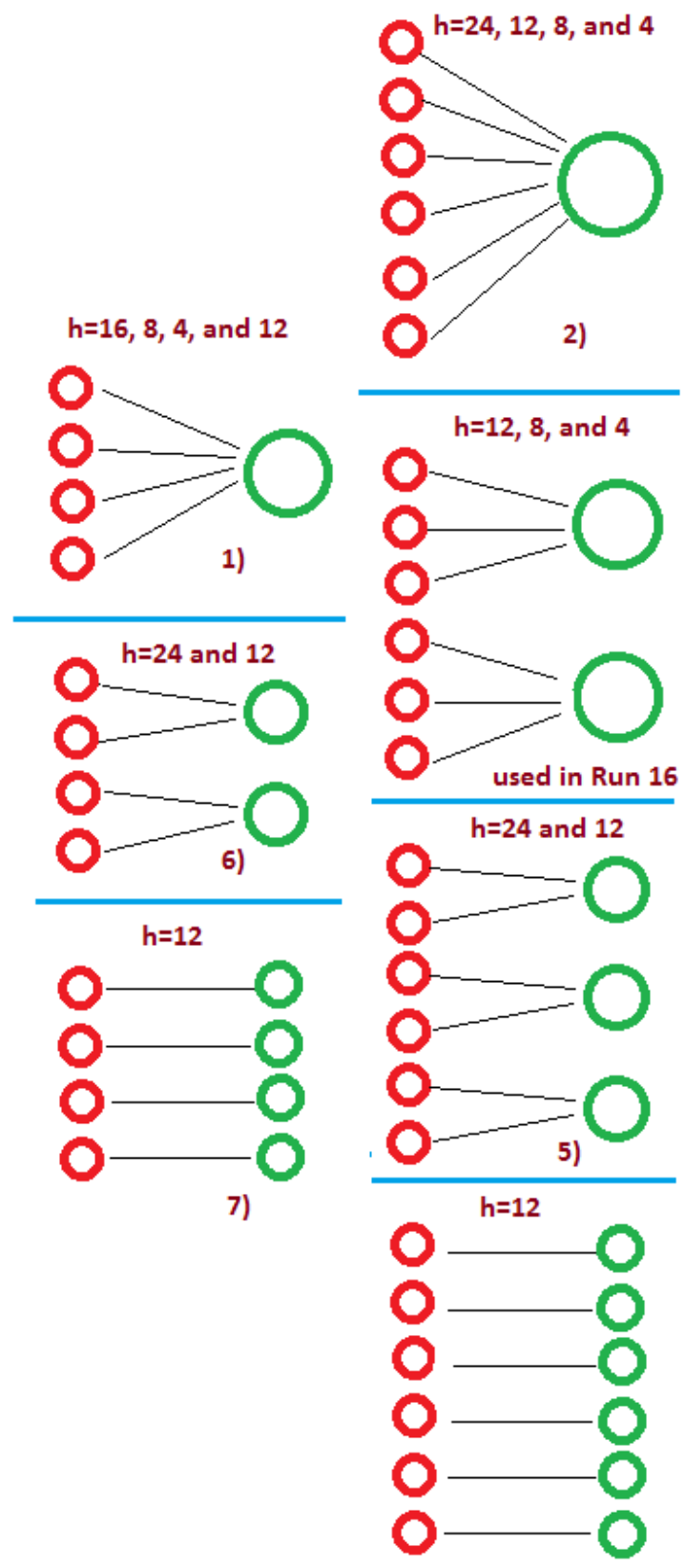

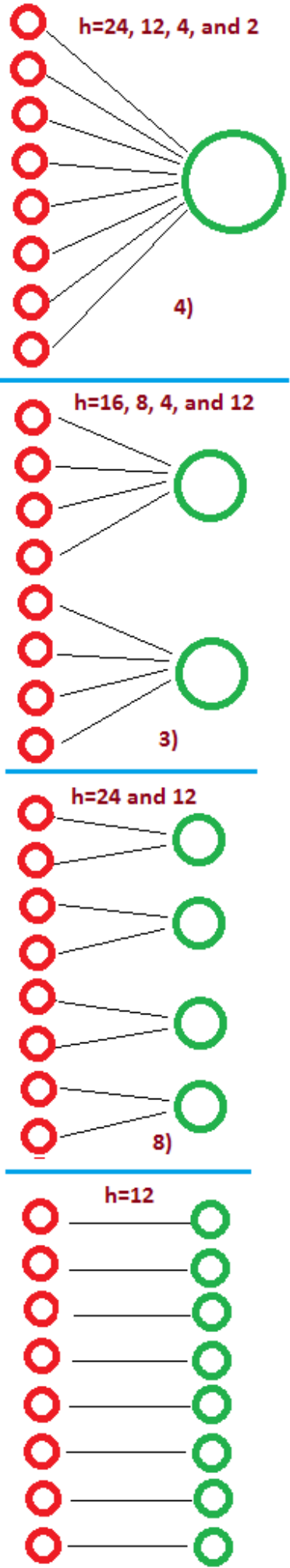

Figure 8: Scenarios discussed in the text. The red circles represent bunches injected into the AGS and the green ones represent bunches at AGS extraction. The numbers next to each scenario refer to the list of scenarios in the text where they are described. Also shown are the AGS Rf harmonics that would be used. All acceleration after the merges uses $h=12$ (except perhaps case 4 , the 8 to 1 merge). 


\section{Summary}

In Run 16 Tandem Au was used to fill RHIC for a brief period. Instead of the 12 pulses used with EBIS only 6 were used and the 12:6 AGS merge was eventually removed because it was hurting machine performance and was unnecessary. A bunch intensity of 2.5e9 ions was eventually reached with a flattop $\varepsilon$ of about $0.59 \mathrm{eV} \cdot \mathrm{s} / \mathrm{n}$ vs. $3.1 \mathrm{e} 9$ ions and $0.75 \mathrm{eV} \cdot \mathrm{s} / \mathrm{n}$ for EBIS $\mathrm{Au}$. The supercycle could have been shortened to about $4.6 \mathrm{sec}$ instead of the $6.0 \mathrm{sec}$ used with EBIS (assuming no related issues with Tandem).

Given the intensity delivered from Tandem, and previous experience with running Tandem Au, if everything was optimal one would have expected a bunch intensity of about 4.1e9 ions instead of 2.5e9 ions. There are several reasons for the difference. First, when the measurement was made, the Tandem intensity was about $24 \mathrm{e} 9$ ions, but it was as high as $30 \mathrm{e} 9$ ions. If the measurement was taken at this higher intensity it would likely have been more like $3.0 \mathrm{e} 9$ since the Booster late intensity was $20 \%$ higher. The Booster efficiency was also not optimal, if it were that would account for another $12 \%$, which would bring the bunch intensity up to about 3.4e9 ions. This is still far from $4.1 \mathrm{e} 9$ ions, and that difference is likely mainly due to the poor quality bunches coming out of the Booster.

A 6:2:1 merge was used instead of a 4:2:1 merge in the Booster because the Tandem beam must be captured into $h=6$ instead of $h=4$ buckets. The $6: 2$ part of that merge doesn't work well (see Figure 2) and appears to be mainly responsible for the poor bunch quality. If the $\varepsilon$ of the bunches injected into the AGS were the same as with EBIS, the flattop $\varepsilon$ would be expected to be more like $1.45 \cdot 0.3 \mathrm{eV} \cdot \mathrm{s} / \mathrm{n}=0.44 \mathrm{eV} \cdot \mathrm{s} / \mathrm{n}$, not $0.59 \mathrm{eV} \cdot \mathrm{s} / \mathrm{n}$.

If Tandem Au is revisited, it might be possible to use a 6:3:1 type merge in the Booster analogous to the one used in the AGS which, at least there, seems to be $\varepsilon$ preserving. If that were successful, bunch intensities close to $4.1 \mathrm{e} 9$ ions and a flattop $\varepsilon$ around $0.44 \mathrm{eV} \cdot \mathrm{s} / \mathrm{n}$ might be achievable. However, since Au31+ is used for Tandem instead of Au32+, the extraction energy is $6 \%$ lower. This could adversely affect the stripping and overall transfer efficiency. The Booster extraction energy could be raised to that used with EBIS, but that energy could be too high for efficient extraction since the F3 kicker might not be strong enough. Alternately, Au32+ could be used from Tandem, though the intensity available for that has been somewhat lower than it is for Au31+. When Tandem Au was run routinely the bunches were not merged in the Booster and it was at this lower extraction energy. In that case the transfer efficiency was as high as it is for the EBIS Au 8:4:2 setup. So, it's not clear how much of a problem the slightly lower extraction energy would really be if the injected $\varepsilon$ is reasonable.

If this 6:3:1 type merge were successful in reducing the injected $\varepsilon$ to EBIS Au levels, the intensity at the end of the AGS injection porch could potentially be 8e9 ions or so. Instabilities on the injection porch were evident even with the EBIS Au cycle where the intensity was in the $6 e 9$ ion range, and they would likely become more of an issue at this higher intensity. There are 
ways of alleviating that problem though, and it still might not be difficult to stop them from happening. The chances of burning a hole in the AGS vacuum chamber with the beam would also increase.

In the preceding section possible scenarios for increasing the bunch intensity further were described. These included using only 4 Tandem pulses which could be merged into one bunch in the AGS and merging all 6 pulses into 1 bunch in the AGS. Even though Tandem has never delivered 8 pulses in a supercycle, the possibility of using 8 pulses to create 2 bunches in the AGS and of merging all 8 injected bunches into 1 was also discussed. Other cases with lower bunch intensity but more bunches at AGS extraction and lower $\varepsilon$ were also described.

It should be noted that although some of these estimates for intensities and emittances look rather impressive, they were only made for setups with a Booster merge that provides the same $\varepsilon$ as the EBIS Au one does and they neglect any possible effects of the lower Booster extraction energy. Given that, the estimates shouldn't be surprising since the extracted intensity per Booster cycle for Tandem Au (when using 4 pulses) has been a factor of 3 higher than it is for EBIS Au. The $\varepsilon$ estimates also assume 45\% growth on the ramp and although measurements last run suggest this to be the case independent of the $\varepsilon$ at the beginning of the ramp, they are only based on data with 3 early ramp emittances $(0.051,0.415$, and $0.52 \mathrm{eV} \cdot \mathrm{s} / \mathrm{n}){ }^{30}$

If the Booster merge worked well, the $\varepsilon$ of the bunches injected into the AGS might be even smaller than it is for EBIS Au because the unmerged Booster bunches using Tandem appear to be significantly smaller than they are when using EBIS. ${ }^{31}$ It's also important to remember that Tandem Au operation is much more tuning intensive and not nearly as stable as EBIS Au operation is.

\footnotetext{
${ }^{30}$ See footnote 15 and also note that the low $\varepsilon$ case is with 10 of the 12 buckets used for acceleration up the ramp filled with relatively low intensity bunches and the other 2 empty. The other 2 cases are with 2 of the h=12 buckets filled with relatively high intensity bunches and baby bunches in the others. It's also true that the small $\varepsilon$ measurement $(0.051 \mathrm{eV} \cdot \mathrm{s} / \mathrm{n})$ was pieced together after the fact. Even though it looks like a valid measurement, if it was smaller than that it would mean that the $\varepsilon$ growth up the ramp would be larger.

${ }^{31}$ See footnote 20 . That observation was not taken into account in the $\varepsilon$ estimates given in this document.
} 